\title{
Tratamiento quirúrgico de las úlceras por presión en el Hospital Universitario Japonés
}

\author{
Dra. Tania Pinto Berrios \\ Cirujana Plástica Reconstructiva del Hospital Universitario Japonés \\ Dr. Edgar Achá \\ Jefe del Servicio de Cirugía Plástica Reconstructiva del Hospital Universitario Japonés
}

\section{RESUMEN}

Se realizó un estudio en el servicio de Cirugía Plástica del Hospital Japonés, que pretende mostrar la experiencia obtenida en el tratamiento quirúrgico de úlceras de presión, para lo cual se revisaron las historias clínicas de todos los pacientes a los que se les efectuó algún tipo de colgajo, para cobertura de úlceras por presión, aplicándose el protocolo antes mencionado por un periodo de 5 años, comprendido entre junio de 2009 y junio de 2015 . Se realizó este trabajo, para que en base a los datos obtenidos se pueda aplicar protocolos de normatización similares a los ya existentes que logren optimizar el manejo de estos pacientes.

PALABRAS CLAVES; Tratamiento quirúrgico, cobertura de úlceras por presión.

\section{ABSTRAC}

A study was done in the service of Plastic Surgery of the Japanese Hospital which aims to show the experience in the surgical treatment of pressure ulcers, for which the medical records of all patients who underwent some kind of flap were reviewed for coverage of pressure sores, applying the protocol above for a period of five years, between June 2009 and June 2015. this work was conducted to that based on the data obtained can be applied to protocols similar standardization existing achieve optimize the management of these patients

KEYWORDS; surgery, pressure ulcers coverage

\section{Introducción.}

Las ulcéras por presión son un problema bastante común en pacientes con paraplejia, o en pacientes con movilidad reducida debido a múltiples factores como el mal manejo intrahospitalario de los pacientes con trauma raquimedular en su fase aguda, mielomeningocele, o pacientes con alteraciones neurológicas que limiten la movilidad, o por la falta de preparación de los mismos para aceptar la situación y adaptarse a ella.

Thomas Rowling fue el primero quien realizó este tipo de descripciones a partir de las lesiones que encontraba en las momias, la biblia también cita a las úlceras de Lázaro en el libro de Isaías.

El mayor adelanto en el tratamiento quirúrgico se lo realizó durante la primera guerra mundial y con el desarrollo de la cirugía comenzaron a utilizarse injertos y colgajos para cubrir estos defectos.

\section{Fisiopatología.}

\section{Clasificación:}

\section{Según su profundidad}

ESTADIO GRADO I Eritema cutáneo que no palidece en piel intacta; La lesión precursora de una ulceración en la piel. En individuos de piel oscura también pueden ser indicadores la decoloración de la piel, calor, edema, induración o insensibilidad.

ESTADIO GRADO II Pérdida parcial del grosor de la piel que puede afectar a epidermis y/o dermis. La úlcera es una lesión superficial que puede tener aspecto de abrasión, flictena, o pequeño cráter superficial.

ESTADIO GRADO III Pérdida total del grosor de la piel, con lesión o necrosis del tejido subcutáneo, pudiéndose exten- 
der hacia dentro pero sin afectar la fascia subyacente. La lesión presenta el aspecto de un cráter que puede socavar o no al tejido subyacente.

ESTADIO GRADO IV Plena lesión de todo el grosor de la piel con destrucción masiva, necrosis tisular o daño en el músculo, hueso o elementos de sostén. Las lesiones de estadio IV pueden presentar trayectos sinuosos y socavados.

\section{Tratamiento quirúrgico}

\section{Protocolo quirúrgico:}

Escisión de los bordes de la herida.

Bursectomía completa.

Resección o abrasión de la prominencia ósea.

Hemostasia.

Cobertura con colgajo bien vascularizado.

\section{Protocolo postquirúrgico:}

Almohadillado importante con apósitos y Tensoplast $®$.

Antibioticoterapia por siete días, coincidiendo con el tiempo de permanencia del drenaje.

Mantenimiento del drenaje siete días mínimo.

Retiro de puntos 14 días mínimo.

\section{Tratamiento rehabilitador:}

Intentar disminuir espasticidad.

En muchas instituciones de nuestro país, este tipo de patología es tratada de forma aislada por diferentes servicios, lo cual hace muy difícil estandarizar el tratamiento y las normativas para lograr así una atención integral.

\section{Materiales y métodos}

Se realizó un estudio de tipo retrospectivo, basado en la revisión de historias clínicas de los pacientes que se realizaron algún tipo de colgajo, para la cobertura de úlceras por presión por parte del Servicio de Cirugía Plástica del Hospital Universitario Japonés, en un periodo de cinco años comprendido entre los meses de junio 2009 a junio de 2015.

Se tomaron variables como por ejemplo edad, sexo, patología de base, tipo de úlcera, uso de cojín antiescaras, tipo de colgajo realizado y complicaciones posteriores, las cuales medimos en relación al número de controles postoperatorios a los que acudió cada paciente.

\section{Resultados}

Se realizaron un total de 23 colgajos en éste periodo, de los cuales fueron $12(52 \%)$ úlceras isquiáticas, $5(21 \%)$ úlceras sacras y 6 (27\%) úlceras trocanterías (Imagen 1).

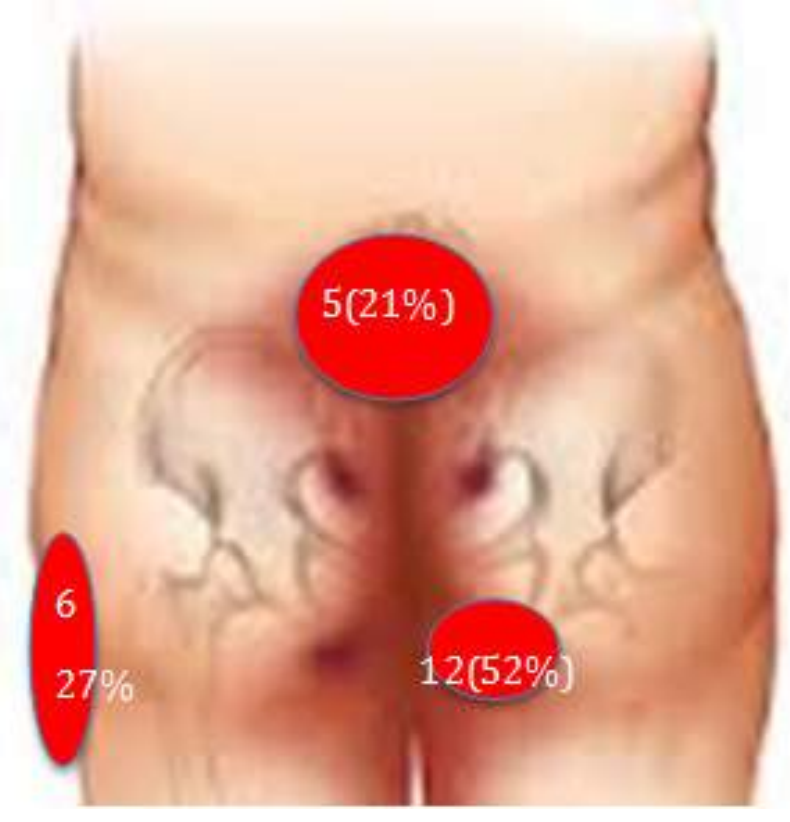

De las úlceras sacras 3 (13\%) fueron colgajos de glúteo mayor en isla, basados en perforantes de la glútea superior (Imagen 2), 1 (4\%) fue con colgajo lumbar con cierre directo y otro fue tratado con colgajos triangulares de avance bilaterales (4\%) (Imagen 3)
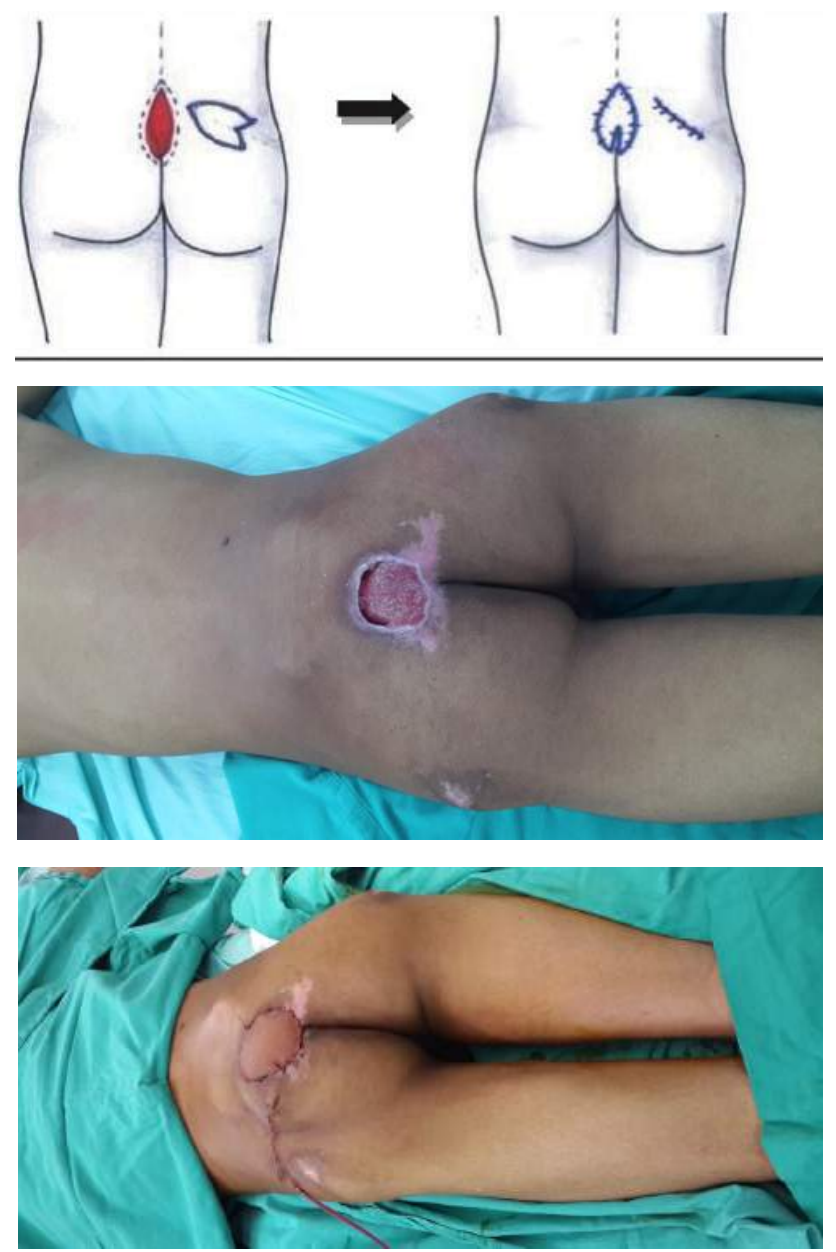


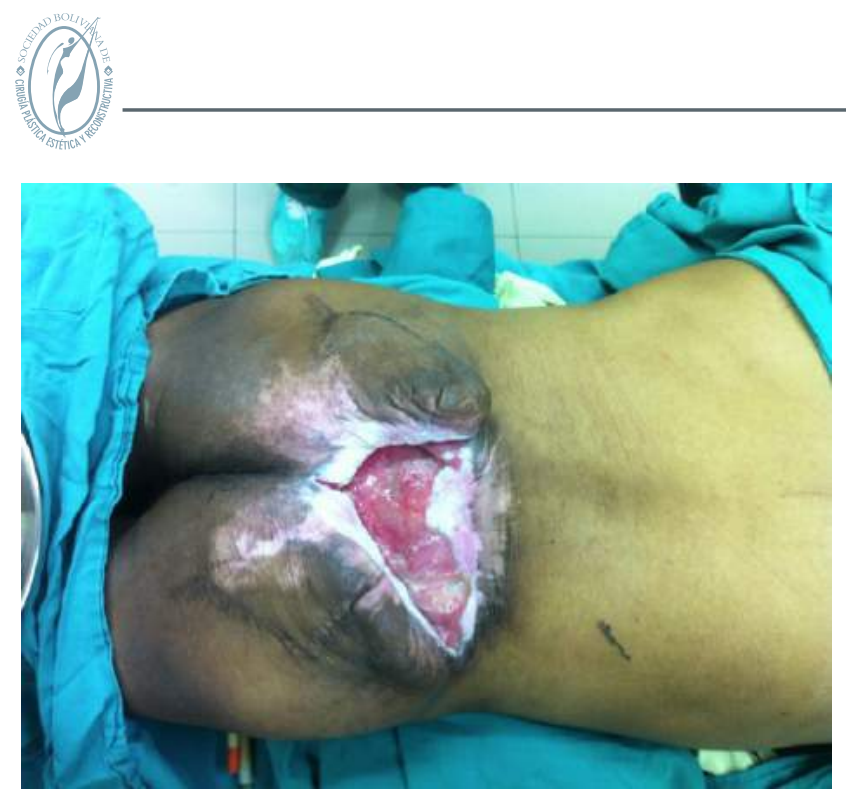

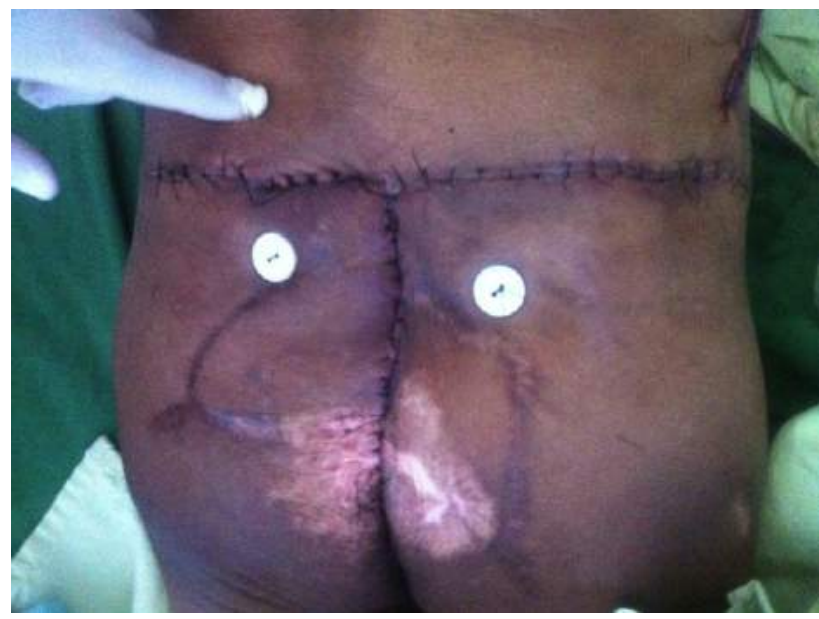

De las úlceras Isquiáticas 11 (45\%) fueron colgajos de glúteo mayor a pedículo inferior con isla de piel distal y cierre de zona dadora, 3 (13\%) fueron colgajos de glúteo mayor a pedículo inferior con isla contigua de piel y cierre en $\mathrm{VY}$ y uno (4\%) fue colgajo de músculos isquiosurales con isla contigua de piel y cierre en VY. (Imagen 4)
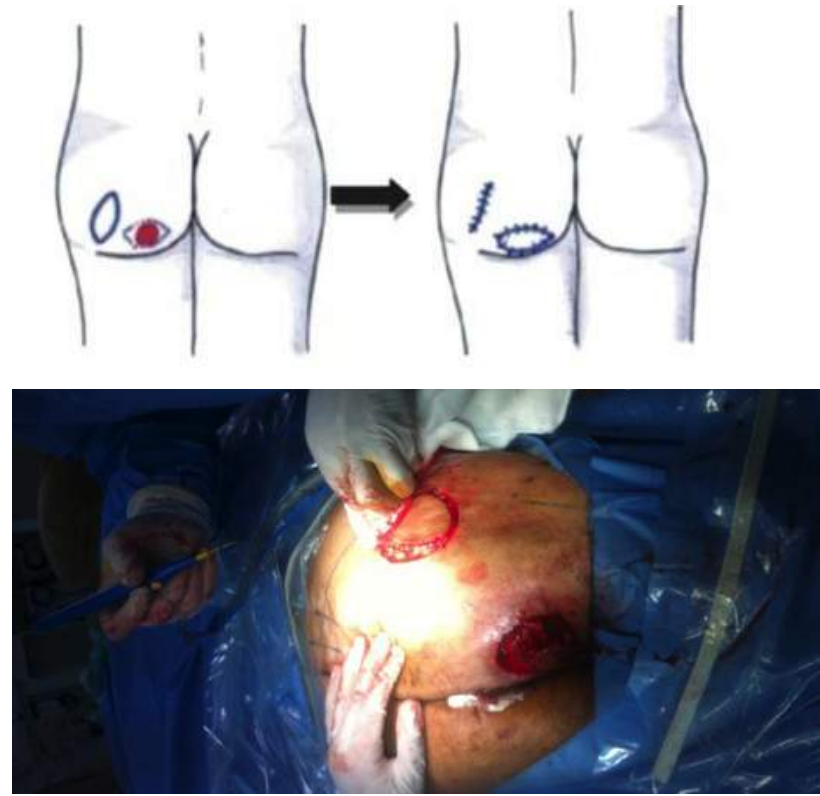
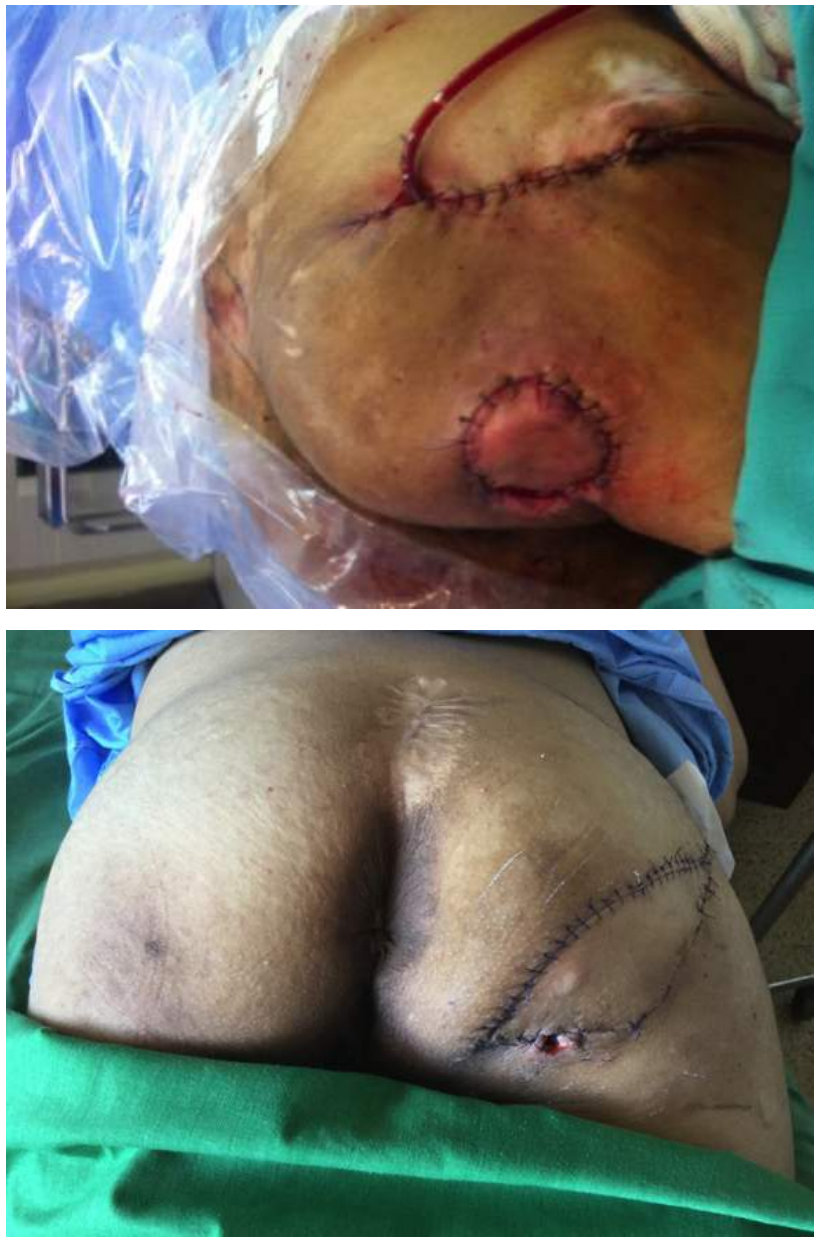

En las úlceras Trocantéricas 5(23\%) fueron colgajo tensor de fascia lata transpuesto a $90^{\circ}$ y 1 ( $4 \%$ ) fue colgajo de fascia lata con pastillas de piel contiguas y cierre en VY.

En cuanto a la edad y sexo fue más frecuente en 18 (78\%) pacientes hombres y 5 (22\%) pacientes mujeres; la edad oscilaba entre los 20 y 60 años con una media de 30 años.

En cuanto al uso de cojines antiescaras, ninguno de los pacientes las usaba, por lo que se indicó antes de la cirugía, pero como es difícil de conseguir en nuestro país apenas 2 (8\%) pacientes pudieron acceder a él.

Las complicaciones inmediatas como congestión venosa, se presentaron en 8 (34\%) pacientes de los cuales 4 (17\%) tuvieron pérdida parcial del colgajo, menor al $5 \%$ de la superficie total cuya resolución fue espontánea y no requirieron de otro tipo de terapéutica y 2 (8\%) tuvieron seroma que se reabsorbió de forma espontánea.

En cuanto a las complicaciones tardías se registró una recidiva la cual se solucionó reavanzando el colgajo pero cabe mencionar que después del retiro de puntos solo 3 (13\%) pacientes acudieron a sus controles posteriores. 

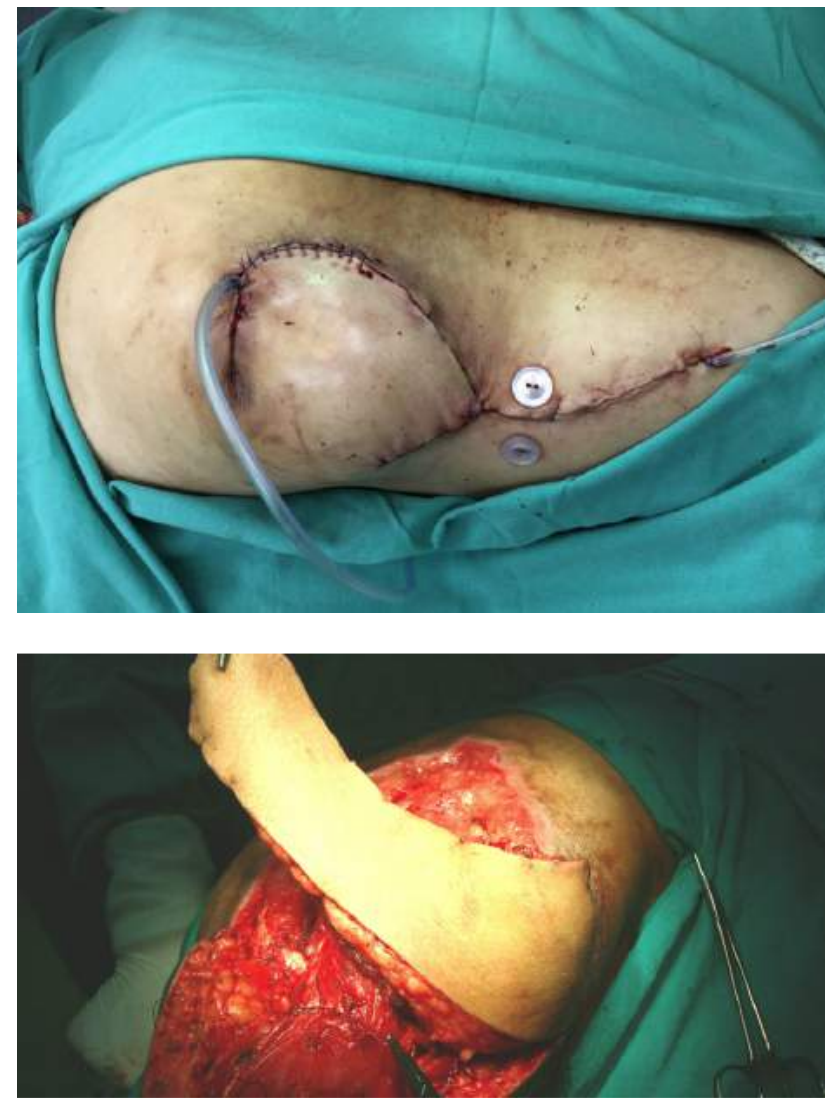

\section{Discusión}

La fisiopatología y manejo es poco conocido por la mayoría de médicos. El impacto de estas lesiones es enorme, en términos de morbilidad y rehabilitación así como gastos hospitalarios.

Es importante mencionar que para la realización de cobertura con colgajos, en todos los casos, se cumplió con los principios de realizar un desbridamiento excisional de la bursa y cualquier calcificación heterotópica, osteotomías en caso de ser necesario y adecuada cobertura para contribuir a la detersión biológica y controlar la infección local.

Aunque no tuvimos recidiva obtenida en este estudio, se debe tener en cuenta que no hubo seguimiento ya que los pacientes en nuestro medio una vez operados acuden solo hasta que se les retira los puntos.

El uso del cojín antiescaras en nuestros pacientes es muy difícil ya que en nuestro medio no se consigue, teniendo los pacientes que intentar conseguirlo en el exterior del país.

Algunos estudios sugieren que en algún momento de la vida, 30\% de pacientes con lesión medular desarrollarán úlceras por presión 1-3. Estas lesiones por presión son algunas de las causas de aumento de estancia hospitalaria por sobreinfecciones y comorbilidades en este tipo de pacientes convirtiéndose en un problema de salud pública.
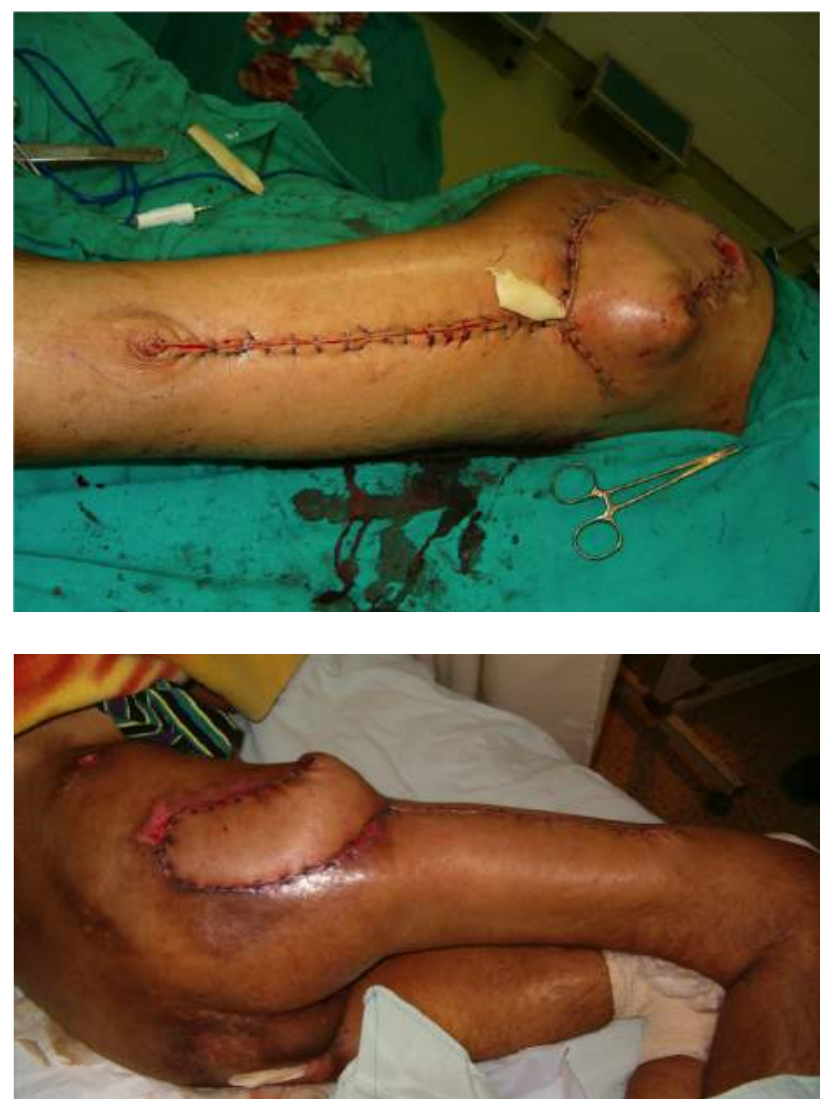

\section{Conclusión}

El manejo de los pacientes con lesiones por presión, requiere un programa de manejo transdiciplinario

Este análisis retrospectivo del manejo quirúrgico que se realiza en el hospital Universitario Japonés, será una herramienta que ofrecerá la posibilidad de evaluar los resultados, corregir, implementar protocolos existentes en otros países y normatizar la atención de estos pacientes.

\section{Bibliografía}

1. Yohena R, Olivero Vila F. Úlceras sacras. Normatización de la selección de colgajos según la forma del defecto. Revista argentina de Cirugía Plástica 2004;1:37-44.

2. Bauer, J. Phillips, LG. Pressure sores: MOC-PSSM CME article. Plast. Reconstr. Surg 2008;121(1):1-10.

3. Garber S, Rintala D. Pressure ulcers in veterans with spinal cord injury: a retrospective study. J Rehabil Res Dev. 2003;40:433-441. 